\title{
Resección de tumor intramedular guiada por fluorescencia con ácido aminolevulínico
}

L.M. Bernal-García; J.M. Cabezudo-Artero; M. Ortega-Martínez; I. Fernández-Portales; J. Giménez-Pando; L.F. Ugarriza-Echebarrieta; J. Mata-Gómez; M. Molina-Orozco y J.F. Malca-Balcázar

Servicio de Neurocirugía. Hospital Universitario Infanta Cristina. Badajoz.

\section{Resumen}

La resección guiada por fluorescencia con ácido aminolevulínico (5-ALA) se ha demostrado útil para el tratamiento quirúrgico de los gliomas cerebrales malignos. También parece útil en el tratamiento de otros tipos de tumores, tanto cerebrales como intramedulares. Presentamos el caso de un paciente con un tumor intramedular en el que la fluorescencia con 5-ALA fue de utilidad para la localización intraoperatoria de la lesión, para la identificación de pequeños nódulos en el parénquima medular y para lograr la exéresis completa del tumor.

PALABRAS CLAVE: Ácido aminolevulínico. 5-ALA. Ependimoma. Fluorescencia. Tumor intramedular.

Fluorescence-guided resection with 5-aminolevulinic acid of an intramedullary tumor

\section{Summary}

Fluorescence-guided resection with 5-aminolevulinic acid (5-ALA) has demonstrated its usefulness in the resection of malignant cerebral gliomas. It also seems useful for the treatment of other types of cerebral and intramedullary neoplasms. We present the case of a patient with an intramedullary tumor in who fluorescence-guided resection was useful for intraoperative localization, definition of small tumor nodules and in order to achieve a complete resection of the tumor.

KEY WORDS: Aminolevulinic acid. 5-ALA. Ependymoma. Fluorescence. Intramedullary neoplasms.

\section{Introducción}

La resección guiada por fluorescencia con ácido aminolevulínico (5-ALA) trata de aprovechar la capaci-

Recibido: 30-06-09. Aceptado: 15-02-10 dad de algunos tejidos tumorales de acumular porfirinas fluorescentes que se hacen visibles en el campo quirúrgico con una coloración diferente al tejido normal cuando se utiliza una luz de una longitud de onda adecuada. Esta técnica está perfectamente establecida para el tratamiento quirúrgico de los gliomas cerebrales malignos ${ }^{1,6-8} \mathrm{y}$, aunque aún no está disponible en muchos centros, está en pleno desarrollo al haber demostrado mejores tasas de resección completa en este tipo de tumores ${ }^{8}$.

Se han publicado casos de otros tipos de tumores en los que la fluorescencia con 5-ALA puede ser útil para conseguir resecciones completas sin dejar restos tumorales que puedan pasar inadvertidos durante la cirugía. Es el caso de meningiomas invasivos ${ }^{3,4}$, metástasis cerebrales ${ }^{9} \mathrm{e}$, incluso, tumores intramedulares ${ }^{1,5}$.

En este trabajo presentamos un caso de tumor intramedular que fue tratado quirúrgicamente en nuestro servicio, en el que la fluorescencia con 5-ALA fue de utilidad para la localización intraoperatoria y la exéresis completa de la lesión.

\section{Caso clínico}

Mujer de 55 años de edad, con clínica de parestesias de 3 años de evolución que comenzaron inicialmente por extremidad superior izquierda, extendiéndose posteriormente a extremidad superior derecha y extremidad inferior izquierda. Año y medio después se añadió debilidad progresiva de extremidades izquierdas, espasmos musculares e incontinencia urinaria. Se realizó una resonancia magnética (RM) cervical que mostró un engrosamiento de la médula a nivel $\mathrm{C} 1-\mathrm{C} 2$ en las secuencias potenciadas en $\mathrm{T} 1$, con captación intensa y homogénea de contraste paramagnético (Figura 1). En las imágenes potenciadas en T2 la lesión se apreció como isointensa, con una hiperintensidad perilesional secundaria a edema que llegaba hasta C5C6. Los hallazgos fueron compatibles con astrocitoma o ependimoma.

Abreviaturas: 5-ALA: ácido aminolevulínico. RM: resonancia magnética. 

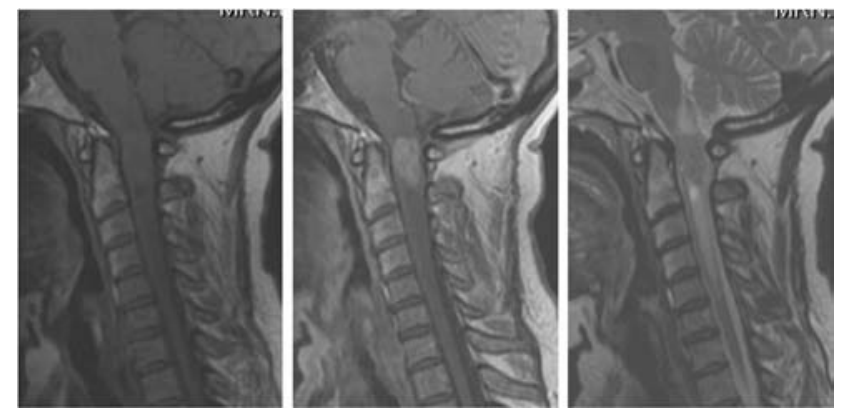

Figura 1. RM cervical, secuencias T1 sin y con contraste y T2: se aprecia una lesión intramedular a nivel C1-C2, isointensa en T1 y T2 y con captación intensa de contraste paramagnético.
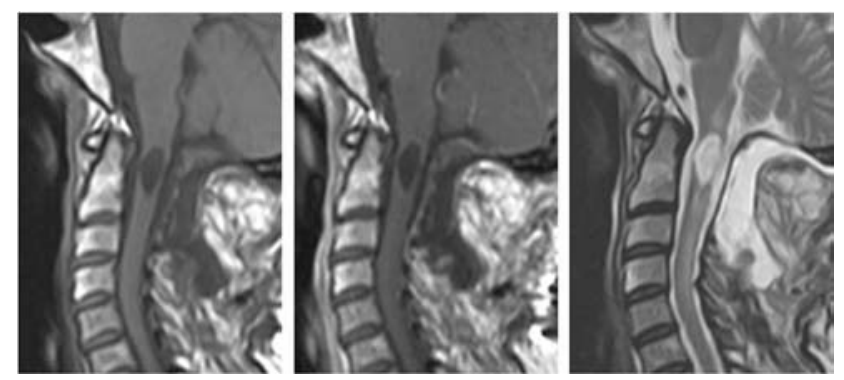

Figura 4. RM cervical postquirúrgica en secuencias T1 sin y con contraste y T2. Se aprecia la cavidad intramedular sin captaciones patológicas.

Se decidió realizar tratamiento quirúrgico. Ante la sospecha de que se tratara de un astrocitoma, se decidió administrar 5-ALA en previsión de que pudiera ser de utilidad.

La paciente fue intervenida quirúrgicamente, previa administración de solución oral con 5-ALA 3 horas antes de la inducción anestésica. No se utilizó monitorización electrofisiológica intraoperatoria al no disponer de la misma en nuestro centro. Se realizó una craniectomía suboccipital, resección del arco posterior de $\mathrm{C} 1$ y de la espinosa y lámina de C2. Tras la introducción del microscopio quirúrgico se procedió a la apertura dural por línea media, apreciándose la médula engrosada y de una coloración normal. Con la luz polarizada se objetivó, por transparencia, una coloración rosada en la médula (Figura 2) que sirvió de guía para localizar la altura a la que realizar la mielotomía por el surco medio posterior. Con la luz blanca se profundizó hasta llegar a la lesión, que presentaba un color violáceo y un plano de clivaje que la separaba del tejido medular sano. Con luz polarizada se apreció de un rojo intenso (Figura 3). Se envió una muestra para estudio histopatológico intraoperatorio que fue informada como ependimoma. Se amplió la mielotomía hasta los límites de la lesión y el tumor se extirpó con el uso de la luz blanca. Cuando parecía que se había conseguido una exéresis completa, con la luz polarizada se descubrieron dos pequeños focos de fluorescencia roja intensa situados en la médula que se extirparon. Se revisó el lecho quirúrgico nuevamente con la luz polarizada, descartando la presencia de fluorescencia.

En el postoperatorio inmediato la paciente presentó un leve empeoramiento neurológico que recuperó posteriormente con tratamiento rehabilitador, quedando con una alteración de la sensibilidad posicional atribuible a la mielotomía.

Una RM postquirúrgica de control (Figura 4) constató la resección completa de la lesión, sin mostrar captaciones

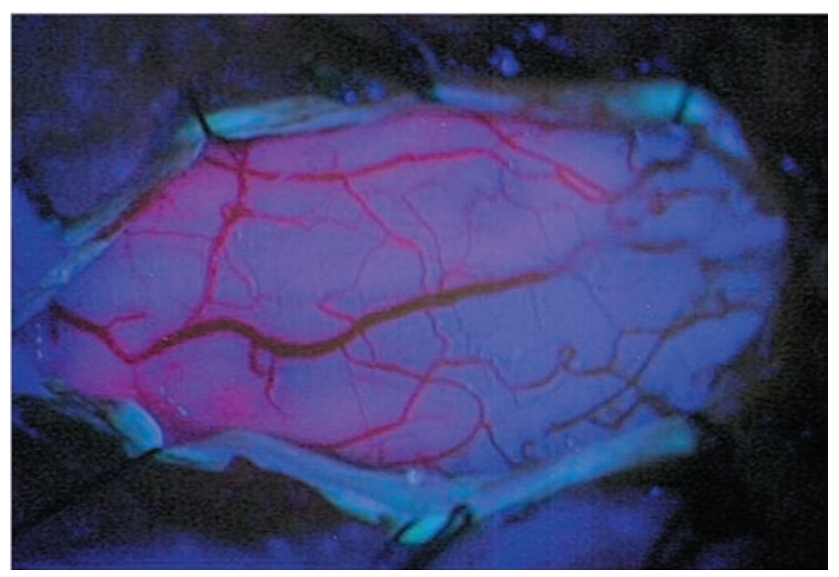

Figura 2. Médula cervical con luz violeta-azul. Se aprecia la fluorescencia rosada por transiluminación.
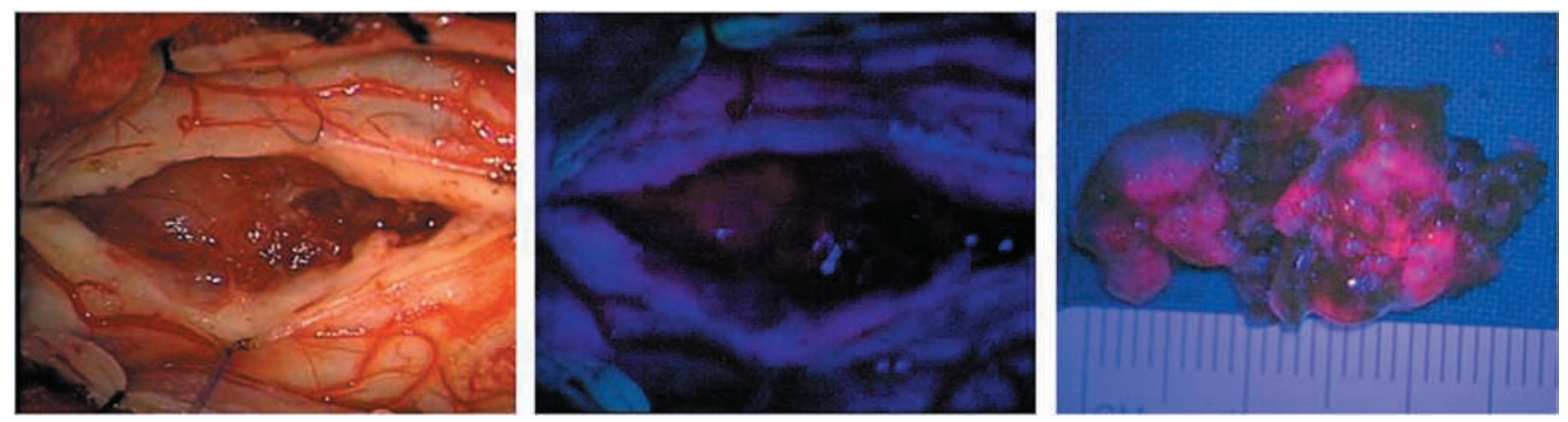

Figura 3. Médula cervical con mielotomía por surco medio posterior. Con luz blanca se aprecia una lesión violácea que emite fluorescencia intensa con luz violeta-azul. 
patológicas.

El informe histopatológico definitivo de la tumoración mostró una lesión moderadamente celular constituida por células de núcleos ovalados y redondos llegando a formar pseudorrosetas. El índice proliferativo fue menor del 1\% y expresaban proteína glial fibrilar ácida. Todo ello compatible con un ependimoma grado II de la OMS.

\section{Discusión}

El 5-ALA es un precursor de la síntesis del grupo hemo. $\mathrm{Su}$ administración exógena por vía oral puede inducir la acumulación de porfirinas fluorescentes en las células tumorales, de manera que cuando el tejido tumoral es iluminado con una luz violeta-azul (longitud de onda de entre 375 y $440 \mathrm{~nm}$ ), se puede apreciar una fluorescencia roja resultado de la excitación de la protoporfirina IX acumulada $^{6-8}$. Esto permite la distinción intraoperatoria del tejido patológico, lo que facilita la resección tumoral y ayuda a conseguir mayores tasas de resección completa al detectar restos tumorales que, sin esta técnica, podrían pasar desapercibidos ${ }^{8}$.

Desde que Stummer et al. ${ }^{6-8}$ publicaran sus resultados demostrando la utilidad del 5-ALA para mejorar el grado de la resección de gliomas cerebrales malignos, la utilización de esta técnica está en franca difusión, habiéndose publicado artículos que hacen referencia a su utilidad en casos de lesiones distintas a los gliomas malignos. En este sentido, Morofuji et al. ${ }^{4}$ y Kajimoto et al. ${ }^{3}$ la han utilizado como ayuda en la resección de meningiomas invasivos y Utsuki et al. para la resección de metástasis cerebrales ${ }^{9}$ y en un caso de hemangioblastoma de cerebelo ${ }^{10}$.

El uso de la fluorescencia para la resección de tumores intramedulares ha sido comunicada previamente por otros autores. Arai et al. ${ }^{1}$ publicaron 3 casos de ependimomas intramedulares en los que la fluorescencia intraoperatoria del tejido tumoral fue de utilidad para conseguir la resección completa de las lesiones. Shimuzu et al..$^{5}$ consiguen una resección completa de un ependimoma intramedular cervical gracias al uso de esta técnica.

El objetivo del tratamiento de los tumores intramedulares debe ser conseguir una resección quirúrgica lo más amplia posible sin añadir déficits neurológicos. Con técnica microquirúrgica la resección es completa en más del $75 \%$ de los $\operatorname{casos}^{2}$. La utilización de la fluorescencia intraoperatoria podría ser de gran ayuda para conseguir resecciones lo más completas posibles sin dañar el tejido medular sano. Pero además, podría ser una herramienta más para la localización intraoperatoria de las lesiones: una vez expuesta la médula puede ser útil para confirmar que la exposición es suficiente y que nos encontramos en el nivel adecuado para comenzar con la mielotomía. En nuestro caso, una vez expuesta la médula y con la luz violeta-azul, pudo comprobarse una coloración rosada que permitió guiar y centrar la extensión de la mielotomía para tener acceso al tumor.

Como apuntan Shimizu et al. ${ }^{5}$ esta técnica podría ser de utilidad para reducir el tumor residual y mejorar las tasas de resección completa de otras lesiones intramedulares primarias.

Existen otras neoplasias del sistema nervioso central, incluso de bajo grado, que pueden emitir fluorescencia en respuesta a la administración exógena del 5-ALA. Se necesitan estudios que intenten dilucidar el motivo por el cual algunos tumores de bajo grado, como el ependimoma intramedular que describimos en este artículo, pueden comportarse igual que los gliomas cerebrales de alto grado.

\section{Conclusiones}

La administración oral de 5-ALA previa a la cirugía y el uso de un microscopio quirúrgico adaptado para detectar fluorescencia tumoral pueden ayudar a la localización intraoperatoria de ependimomas intramedulares y guiar la extensión de la mielotomía necesaria para la exéresis tumoral, así como para conseguir extirpar los restos tumorales que pudieran quedar en el lecho quirúrgico.

Quizás esta técnica podría ser de utilidad en otros tipos de lesiones intramedulares primarias para su localización intraoperatoria y conseguir resecciones más amplias sin dañar el tejido medular sano circundante.

\section{Bibliografía}

1. Arai, T., Tani, S., Isoshima, A., et al.: Intraoperative photodynamic diagnosis for spinal ependymoma using 5 -aminolevulinic acid: technical note. No Shinkei Geka 2006 Aug; 34: 811-817.

2. Gelabert González, M.: Tumores medulares primarios. Análisis de una serie de 168 pacientes. Rev Neurol 2007; 44: 269-274.

3. Kajimoto, Y., Kuroiwa, T., Miyatake, S.I., et al.: Use of 5-aminolevulinic acid in fluorescence-guided resection of meningioma with high risk of recurrence Case report. J. Neurosurg 2007; 106: 1070-1074.

4. Morofuji, Y., Matsuo, T., Hayashi, Y., Suyama, K., Nagata, I.: Usefulness of Intraoperative photodynamic diagnosis using 5-aminolevulinic acid for meningiomas with cranial invasion: technical case report. Neurosurgery 2008 [ONS Suppl 1] 62:102-104.

5. Shimizu, S., Utsuki, S., Sato, K., Oka, H., Fujii, K., Mii, K.: Photodynamic diagnosis in surgery for spinal ependymoma. Case illustration. J. Neurosurg Spine 2006; 5: 380.

6. Stummer, W., Stocker, S., Wagner, S., et al: Intraoperative detection of malignant gliomas by 5 -aminolevulinic 
acid-induced porphyrin fluorescence. Neurosurgery 1998; 42: $518-526$.

7. Stummer, W., Novotny, A., Stepp, H., Goetz, C., Bise, K., Reulen, H.J.: Fluorescence-guided resection of glioblastoma multiforme by using 5-aminolevulinic acidinduced porphyrins: a prospective study in 52 consecutive patients. J Neurosurg 2000; 93: 1003-1013.

8. Stummer, W., Pichlmeier, U., Meinel, T., Wiestler, O.D., Zanella, F., Reulen, H.J.: Fluorescence-guided surgery with 5aminolevulinic acid for resection of malignant glioma: a randomised controlled multicentre phase III trial. Lancet Oncol 2006; 7: 392-401.

9. Utsuki, S., Miyoshi, N., Oka, H., et al.: Fluorescenceguided resection of metastatic brain tumors using a 5-aminolevulinic acid-induced protoporphyrin IX: pathological study. Brain Tumor Pathol 2007; 24: 53-55.

\section{Comentario al trabajo Resección de tumor intramedular guiada por fluorescencia con ácido aminolevulínico de Bernal García y cols.}

Bernal García y cols. presentan el caso de un ependimoma medular cervical intervenido con la ayuda de fluorescencia intraoperatoria con 5-ALA. Se trata de un caso interesante, dado que la fluorescencia intraoperatoria con 5-ALA es una técnica no disponible todavía en muchos centros y en proceso de desarrollo. Los autores exponen la utilidad de la misma para la identificación de la lesión en la médula tras la apertura dural, para la selección del lugar idóneo donde efectuar la mielotomía, y durante la exéresis tumoral. Aunque su utilización se ha centrado fundamentalmente en la cirugía de los gliomas malignos cerebrales, otras aplicaciones van siendo progresivamente descritas. Si bien son escasos hasta la fecha los casos publicados en la literatura de utilización de 5ALA para la resección de tumores medulares, los autores hacen referencia a los mismos.

Existen sin embargo dos aspectos, que cabe puntualizar. En primer lugar, la resección guiada por fluorescencia con 5-ALA fue descrita para el tratamiento quirúrgico de los gliomas malignos. El 5-ALA es un precursor natural de la hemoglobina a partir del cual se sintetizan y acumulan porfirinas fluorescentes en los tejidos. Estas porfirinas fluorescentes pueden visualizarse intraoperatoriamente con el microscopio quirúrgico modificado. Es precisamente su síntesis intratumoral lo que diferencia al 5-ALA de otras sustancias fluorescentes como la fluoresceína,
10. Utsuki, S., Oka, H., Sato, K., Shimizu, S., Suzuki, S., Fujii, K.: Fluorescence diagnosis of tumor cells in hemangioblastoma cysts with 5-aminolevulinic acid. Case report. J Neurosurg 2010;112:130-132.

Bernal-García, L.M.; Cabezudo-Artero, J.M.; Ortega-Martínez, M.; Fernández-Portales, I.; Giménez-Pando, J.; Ugarriza-Echebarrieta, L.F.; Mata-Gómez, J.; Molina-Orozco, M.; Malca-Balcázar, J.F.: Resección de tumor intramedular guiada por fluorescencia con ácido aminolevulínico. Neurocirugía 2010; 21: 212-216.

Correspondencia: Dr. Luis Miguel Bernal García. Servicio de Neurocirugía. Hospital Universitario Infanta Cristina. Av. de Elvas s/n. 06006 Badajoz.

E-mail: lumi_bega@hotmail.com

que se han empleado igualmente para discriminar el tejido tumoral, pero que penetran en el tumor simplemente por alteración de la barrera hematoencefálica. Este hecho que supone una clara ventaja en cuanto a la especificidad del 5-ALA, supone al mismo tiempo una limitación para su uso en la cirugía de los gliomas de bajo grado. Existe incluso en la actualidad evidencia demostrada de que en el seno de gliomas grado III de la OMS no captantes de contraste, pueden observarse áreas parcheadas de fluorescencia. Sin embargo, esto no parece suceder en los gliomas grado $\mathrm{II}^{1,3}$, no existiendo hasta la fecha, evidencia clara de la utilidad y fiabilidad de la resección guiada por fluorescencia intraoperatoria con 5-ALA en la exéresis de los mismos ${ }^{2}$. El papel del 5-ALA en la cirugía de los gliomas de bajo grado es todavía controvertido, y requiere por tanto de evaluación en mayor profundidad. Curiosamente, el resultado anatomopatológico del caso que nos presentan los autores fue ependimoma grado II de la OMS.

En segundo lugar, resaltar la importancia de que las resecciones quirúrgicas de los gliomas medulares se efectúen con control de monitorización neurofisiológica intraoperatoria. Resultaría de gran interés conocer en el caso que nos ocupa, si la extirpación de las zonas de aspecto normal bajo la luz blanca convencional del microscopio, pero fluorescentes con la luz polarizada, originó cambios 
en la neuromonitorización dado que, tal como exponen los autores, estas áreas del lecho quirúrgico son teóricamente parénquima medular con infiltración tumoral sólo visible con 5-ALA. El objetivo del tratamiento quirúrgico de los tumores medulares debe ser la máxima resección posible persiguiendo la mejoría o al menos la preservación de la función neurológica del paciente. En este sentido, los potenciales evocados somestésicos y motores permiten monitorizar la integridad funcional de los cordones posteriores y del tracto córtico-espinal resultando de gran ayuda durante la resección. Si la utilización de la fluorescencia intraoperatoria en la cirugía de los tumores medulares permitirá alcanzar mayor tasa de resecciones completas sin dañar el tejido medular sano, y por tanto sin un incremento de la morbilidad postoperatoria está todavía por demostrar.

\section{Bibliografía}

1. Ewelt, C., Stummer, W., Klink, B., Felsberg, J., Steiger, H-J., Sabel, M.: Cordectomy as final treatment option for diffuse intramedullary malignant glioma using 5-ALA fluorescence-guided resection. Clin Neurol Neurosurg 2010 (en prensa) doi:10.1016/j.clineuro.2009.12.013.

2. Stockhammer, F., Misch, M., Horn, P., Koch, A., Fonyuy, N., Plotkin, M.: Association of F18-fluoro-ethyl-tyrosin uptake and 5-aminolevulinic acid-induced fluorescence in gliomas. Acta Neurochir 2009; 151: 1377-1383.

3. Widhalm, G., Wolfsberger, S., Minchev, G., et al.: 5Aminolevulinic acid is a promising marker for detection of anaplastic foci in diffusely infiltrating gliomas with nonsignificant contrast enhancement. Cancer 2010 (en prensa) doi: 10.1002/cncr.24903. 